\title{
Pricing Spread Options with Stochastic Interest Rates
}

\author{
Yunguo Jin and Shouming Zhong \\ School of Mathematical Sciences, University of Electronic Science and Technology of China, Chengdu, Sichuan 611731, China \\ Correspondence should be addressed to Yunguo Jin; yunguoj@163.com
}

Received 13 July 2014; Revised 14 October 2014; Accepted 15 October 2014; Published 13 November 2014

Academic Editor: Xuejun Xie

Copyright (C) 2014 Y. Jin and S. Zhong. This is an open access article distributed under the Creative Commons Attribution License, which permits unrestricted use, distribution, and reproduction in any medium, provided the original work is properly cited.

\begin{abstract}
Although spread options have been extensively studied in the literature, few papers deal with the problem of pricing spread options with stochastic interest rates. This study presents three novel spread option pricing models that permit the interest rates to be random. The paper not only presents a good approach to formulate spread option pricing models with stochastic interest rates but also offers a new test bed to understand the dynamics of option pricing with interest rates in a variety of asset pricing models. We discuss the merits of the models and techniques presented by us in some asset pricing models. Finally, we use regular grid method to the calculation of the formula when underlying stock returns are continuous and a mixture of both the regular grid method and a Monte Carlo method to the one when underlying stock returns are discontinuous, and sensitivity analyses are presented.
\end{abstract}

\section{Introduction}

Spread options are financial instruments whose payoffs depend on the price difference of two underlying assets and are widely traded in a range of financial markets. For example, the price spread between heating oil and crude oil (crack spread) represents the value of production (including profit) for a refinery firm. In particular, to energy trading, the derivative contracts are a risk mitigation tool of crucial importance. Carmona and Durrleman [1] presented a general overview of the common features of all spread options by discussing in detail their roles as speculation devices and risk management tools. Their review concentrated on the mathematics of the pricing and hedging of spread option. Hurd and Zhou [2] showed a numerical integration method for computing spread options in two or higher dimensions using the FFT. Tt is based on square integrable integral formulas for the payoff function and, like those methods, it is applicable to a variety of spread option payoff in any model for which the characteristic function of the joint return process is given analytically. Caldana and Fusai [3] proposed a new accurate method for pricing European spread options and obtained a lower bound, as in Bjerksund and Stensland [4], but for general processes. Lo [5] applied the idea of WKB method to provide a simple derivation of Kirks approximation and discussed its validity.
Although spread options [1-9] have come into the limelight in recent years, little research has been published on the pricing of spread options with stochastic interest rates. For options on bonds and other interest-rate-dependent instruments, movements in the interest rate are critical and the assumption of a constant interest rate is inappropriate. In this case, stochastic interest rate may significantly affect the prices.

The pricing of options in the presence of stochastic interest rates can generally be difficult. However, as was discussed in Shreve [10], the choice of another numéraire (e.g., a stochastic bond price) may be more convenient for option pricing in a model with stochastic interest rate. As was pointed out in Kwok [11], a model can be complicated or simple, depending on the choice of the numéraire for the model. Merton [12] used a bond maturing on the option expiration date to hedge European options. Jamshidian [13] observed that the forward price of an asset is its price when denominated in the numéraire of the zero-coupon bond maturing at the delivery date. Geman et al. [14] presented a generalized Black-Scholes-Merton option pricing formula that permits the interest rate to be random.

However, sometimes it is inappropriate to assume that the volatility of the discounted bond price is a constant. There is no reason why interest rates should behave like stock prices. Interest rates certainly do not exhibit the long-term exponential growth seen in the equity markets. 
On the other hand, mean-reverting models are used for modeling a random variable that is not going anywhere. That is why they are often used for interest rates. The Vasicek model takes the following form:

$$
d R(t)=(a-b R(t)) d t+\sigma d W(t)
$$

where $a, b$, and $\sigma$ are positive constants and $W(t)$ is a onedimensional Brownian motion. The model is mean-reverting to a constant level, which is a good property. It is easy to obtain the solution so that there are explicit formulae for many interest rate derivatives. Thus, it is desirable to have a model that combines the strengths of the existing methods.

Furthermore, the technique of measure changes is useful in a variety of asset pricing models. However, few papers focus on the discussion of the merits for it and its applications.

Credit risk arises from the possibility that borrowers and counterparties in derivatives transactions may default. Most financial institutes devote considerable resources to the measurement and management of credit risk. As was pointed out in Hull [15], the credit exposure on a derivatives transaction is more complicated than that on a loan. This is because the claim that will be made in the event of a default is more uncertain. Klein [16] presented an improved method of pricing vulnerable Black-Scholes options under assumptions which were appropriate in many business situations. An analytic pricing formula is derived which allowed not only for correlation between the option's underlying asset and the credit risk of the counterparty but also for the option writer to have other liabilities. Yang et al. [17] extended the model of Klein [16] and considered the pricing of vulnerable options when the underlying asset follows a stochastic volatility model. They used multiscale asymptotic analysis to derive an analytic approximation formula for the price of the vulnerable options and studied the stochastic volatility effect on the option price.

This study aims to fill the gaps and presents three novel spread options pricing models that permit the interest rates to be random. We assume that a discounted zero-coupon bond follows a stochastic process. The volatility of the discounted bond price is a function of time $t$ rather than a constant. The study presents the technique of measure changes to the computations of the spread options. We discuss the excellent feature for the models and techniques presented by us in some asset pricing.

The organization of the remaining part is as follows. Section 2 outlines a spread option pricing model with stochastic interest rates and presents a technique of measure changes to the price of the spread option. Section 3 extends this analysis to jump diffusion model. Section 4 extends this analysis to a vulnerable spread option. Section 5 presents numerical analysis. The final section gives concluding remarks.

\section{Spread Option with Stochastic Interest Rates}

This section constructs a spread option pricing model that permits the interest rate to be random. A key point in the model is to assume that the volatility of the discounted bond price is a function of time $t$ rather than a constant. Using the technique of measure changes, we analyze the price of the spread option when underlying stock returns are continuous. We discuss the excellent features for the model and the technique in some asset pricing models.

2.1. Problem Formulation. Let $(\Omega, \mathscr{F}, \widetilde{\mathbb{P}})$ be a probability space on which is defined a three-dimensional Brownian motion $\widetilde{W}(t)=\left(\widetilde{W}_{a}(t), \widetilde{W}_{b}(t), \widetilde{W}_{c}(t)\right), 0 \leq t \leq T$, and let $\widetilde{\mathbb{P}}$ denote the risk neutral probability measure. In particular, $\widetilde{W}_{a}(t), \widetilde{W}_{b}(t)$, and $\widetilde{W}_{c}(t)$ are independent Brownian motions. Let $\mathscr{F}(t), 0 \leq t \leq T$, be the filtration generated by the Brownian motion. We assume that the interest rate process $R(t)$ is adapted and define a discount process $D(t)=$ $e^{-\int_{0}^{t} R(u) d u}$. Consider a zero-coupon bond that pays 1 unit of currency at maturity $T$. According to the risk-neutral pricing formula, the value of this bond at time $t \in[0, T]$ is $B(t, T)=$ $(1 / D(t)) \widetilde{\mathbb{E}}[D(T) \mid \mathscr{F}(t)]$. Let $S_{1}(t)$ and $S_{2}(t)$ be two stock price processes. The evolutions of the discounted prices will be modeled by the following stochastic differential equations:

$$
\begin{aligned}
& d\left(D(t) S_{i}(t)\right)=D(t) S_{i}(t) v_{i} \cdot d \widetilde{W}_{i}(t), \quad i=1,2, \\
& d(D(t) B(t, T))=D(t) B(t, T) v_{3}(t, T) \cdot d \widetilde{W}_{3}(t),
\end{aligned}
$$

where $\widetilde{W}_{1}(t)=\left(\widetilde{W}_{a}(t), 0,0\right), \widetilde{W}_{2}(t)=\left(\widetilde{W}_{a}(t), \widetilde{W}_{b}(t), 0\right)$, and $\widetilde{W}_{3}(t)=\left(\widetilde{W}_{a}(t), \widetilde{W}_{b}(t), \widetilde{W}_{c}(t)\right), v_{1}=\left(v_{11}, 0,0\right), v_{2}=\left(v_{21}, v_{22}\right.$, $0)$, and $v_{3}(t, T)=\left(v_{31}(t, T), v_{32}(t, T), v_{33}(t, T)\right)$ are three vector volatility processes. The value at time $t \in[0, T]$ of a spread option, expiring at time $T$ with strike price $K$, is

$$
V(t)=\widetilde{\mathbb{E}}\left[e^{-\int_{t}^{T} R(u) d u}\left(S_{1}(T)-S_{2}(T)-K\right)^{+} \mid \mathscr{F}(t)\right] .
$$

\subsection{Pricing the Spread Option}

Definition 1. One defines three new measures.

(1) Suppose one takes the asset price $S_{i}(t), i=1,2$, to be the numéraire. One defines the risk-neutral measure for this numéraire by

$$
\widetilde{\mathbb{P}}^{S_{i}}(A)=\frac{1}{S_{i}(0)} \int_{A} D(T) S_{i}(T) d \widetilde{\mathbb{P}},
$$

for all $A \in \mathscr{F}(T)$.

(2) Let $T$ be a fixed maturity date. One defines the $T$ forward measure $\widetilde{\mathbb{P}}^{T}$ by

$$
\widetilde{\mathbb{P}}^{T}(A)=\frac{1}{B(0, T)} \int_{A} D(T) d \widetilde{\mathbb{P}}
$$

for all $A \in \mathscr{F}(T)$.

Let $\left(\Omega_{2}, \mathscr{F}_{2}, \mathbb{P}_{2}\right)$ be a probability space on which is defined a multidimensional Brownian motion $W(t)=$ $\left(W_{1}(t), W_{2}(t), \ldots, W_{d}(t)\right), 0 \leq t \leq T$. Let $\mathscr{F}_{2}(t), 0 \leq t \leq T$, be the filtration generated by the Brownian motion. The proof of Lemma 2 is like that of the one-dimensional Girsanov Theorem 5.2.3 in [10], except it uses a $d$-dimensional version of Lévy's Theorem. 
Lemma 2 (Girsanov, multiple dimensions, [10]). Let $\Theta(t)=$ $\left(\Theta_{1}(t), \Theta_{2}(t), \ldots, \Theta_{d}(t)\right)$ be a d-dimensional adapted process. Define

$$
\begin{aligned}
& Z(t)=\exp \left\{-\int_{0}^{t} \Theta(u) \cdot d W(u)-\frac{1}{2} \int_{0}^{t}\|\Theta(u)\|^{2} d u\right\}, \\
& \bar{W}(t)=W(t)+\int_{0}^{t} \Theta(u) d u,
\end{aligned}
$$

and assume that

$$
\mathbb{E} \int_{0}^{t}\|\Theta(u)\|^{2} Z^{2}(u) d u<\infty
$$

Then, under the probability measure $\overline{\mathbb{P}}$ given by

$$
\overline{\mathbb{P}}(A)=\int_{A} Z(\omega) d \mathbb{P}(\omega), \quad \forall A \in \mathscr{F}_{2}(T),
$$

the process $\bar{W}(t)$ is a d-dimensional Brownian motion.

For the following Lemma 3, we refer the reader to Theorem 4.3.1 in [10] for its discussion.

Lemma 3 (see [10]). Let $W_{0}(t)$ be a Brownian motion and let $\Delta(t)$ be an adapted stochastic process that satisfies $\mathbb{E} \int_{0}^{T} \Delta^{2}(t) d t<\infty$. Then, $\int_{0}^{t} \Delta(u) d W_{0}(u)$ is a martingale.

Proposition 4. Under a new measure, the following price dynamics holds.

(1) Under the $T$-forward measure $\widetilde{\mathbb{P}}^{T}$, the process $S_{i}(t) / B(t, T), i=1,2$, is a martingale. Moreover,

$$
d\left(\frac{S_{i}(t)}{B(t, T)}\right)=\frac{S_{i}(t)}{B(t, T)}\left(v_{i}-v_{3}(t, T)\right) \cdot d \widetilde{W}^{T}(t),
$$

where $\widetilde{W}^{T}(t)=\left(\widetilde{W}_{a}(t), \widetilde{W}_{b}(t), \widetilde{W}_{c}(t)\right)-\int_{0}^{t} v_{3}(u, T) d u$ is a three-dimensional Brownian motion under $\widetilde{\mathbb{P}}^{T}$.

(2) Under the measures $\widetilde{\mathbb{P}}^{S_{i}}, i=1,2$, the processes $B(t, T) / S_{i}(t)$ and $S_{j}(t) / S_{i}(t), j=1,2, i \neq j$, are $a$ martingale. Moreover,

$$
\begin{gathered}
d\left(\frac{B(t, T)}{S_{i}(t)}\right)=\frac{B(t, T)}{S_{i}(t)}\left(v_{3}(t, T)-v_{i}\right) \cdot d \widetilde{W}^{S_{i}}(t), \\
d\left(\frac{S_{j}(t)}{S_{i}(t)}\right)=\frac{S_{j}(t)}{S_{i}(t)}\left(v_{j}-v_{i}\right) \cdot d \widetilde{W}^{S_{i}}(t),
\end{gathered}
$$

where $\widetilde{W}^{S_{i}}(t)=\left(\widetilde{W}_{a}(t), \widetilde{W}_{b}(t), \widetilde{W}_{c}(t)\right)-v_{i} t$ is a threedimensional Brownian motion under $\widetilde{\mathbb{P}}^{S_{i}}$.

The proof of Proposition 4 will be given in Appendix A.

We need some notations and denote by $\|\cdot\|$ the Euclidean norm in $\mathbb{R}^{3}$. Let

$$
\begin{gathered}
A_{i 3}(t)=\frac{S_{i}(t)}{B(t, T)} \exp \left\{-\frac{1}{2} \int_{t}^{T}\left\|\left(v_{i}-v_{3}(u, T)\right)\right\|^{2} d u\right\}, \\
B_{i 3}(t)=\sqrt{\int_{t}^{T}\left\|v_{i}-v_{3}(u, T)\right\|^{2} d u,}
\end{gathered}
$$

$$
\begin{gathered}
A_{3 i}(t)=\frac{B(t, T)}{S_{i}(t)} \exp \left\{-\frac{1}{2} \int_{t}^{T}\left\|\left(v_{3}(u, T)-v_{i}\right)\right\|^{2} d u\right\}, \\
B_{3 i}(t)=\sqrt{\int_{t}^{T}\left\|v_{3}(u, T)-v_{i}\right\|^{2} d u,} \\
A_{i j}(t)=\frac{S_{i}(t)}{S_{j}(t)} \exp \left\{-\frac{1}{2} \int_{t}^{T}\left\|v_{i}-v_{j}\right\|^{2} d u\right\} \\
B_{i j}(t)=\left\|v_{i}-v_{j}\right\| \sqrt{T-t}, \\
\rho_{1}=\frac{\left(v_{2}-v_{1}\right) \cdot \sqrt{\int_{t}^{T}\left(v_{3}(u, T)-v_{1}\right)^{2} d u}}{\left\|v_{2}-v_{1}\right\|\left\|\sqrt{\int_{t}^{T}\left(v_{3}(u, T)-v_{1}\right)^{2} d u}\right\|}, \\
\rho_{3}=\frac{\left(v_{1}-v_{2}\right) \cdot \sqrt{\int_{t}^{T}\left(v_{3}(u, T)-v_{2}\right)^{2} d u}}{\| \sqrt{\int_{t}^{T}\left(v_{1}-v_{3}(u, T)\right)^{2} d u \|}}, \\
\rho_{2}^{T}\left(v_{1}-v_{3}(u, T)\right)^{2} d u \cdot \sqrt{\int_{t}^{T}\left(v_{2}-v_{3}(u, T)\right)^{2} d u}
\end{gathered} .
$$

For spread options, there is a long history of approximation methods for the computation of the prices. The challenge in pricing spread options stems from the fact that there is no explicit solution. Furthermore, the pricing of options with stochastic interest rates can generally be difficult. Therefore, pricing spread options with stochastic interest rates is a challenging task in finance and is important for both theory and applications.

Theorem 5. The price of the spread option, assuming the riskneutral dynamics given in (2) and (3), is $V(t)=V_{1}(t)+V_{2}(t)+$ $V_{3}(t)$ with

$$
\begin{aligned}
V_{1}(t)= & S_{1}(t) \\
& \times \operatorname{Pr}\left(1-A_{21}(t) e^{B_{21}(t) \xi_{11}}-K A_{31}(t) e^{B_{31}(t) \xi_{12}}>0\right), \\
V_{2}(t)= & -S_{2}(t) \\
& \times \operatorname{Pr}\left(A_{12}(t) e^{B_{12}(t) \xi_{21}}-1-K A_{32}(t) e^{B_{32}(t) \xi_{22}}>0\right), \\
V_{3}(t)= & -K B(t, T) \\
& \times \operatorname{Pr}\left(A_{13}(t) e^{B_{13}(t) \xi_{31}}-A_{23}(t) e^{B_{23}(t) \xi_{32}}-K>0\right),
\end{aligned}
$$

where the random variables $\xi_{i 1}$ and $\xi_{i 2}, i=1,2,3$, have the bivariate standard normal distribution with correlation $\rho_{i}$. For event $\Lambda$, a number $\operatorname{Pr}(\Lambda)$ indicates the probability that $\Lambda$ will occur.

The proof of Theorem 5 will be given in Appendix B. The delta of an option or a portfolio of options is the sensitivity of 
the option or portfolio to the underlying. The sensitivities of the spread option price to initial stock prices $S_{i}, i=1,2$, and spread $K$ are given by $\Delta_{1}=\partial V / \partial S_{1}, \Delta_{2}=\partial V / \partial S_{2}$, and $\kappa=$ $\partial V / \partial K$. The simplest way to calculate the delta, the gamma, and the theta of an option using Monte Carlo simulation is to estimate the option value twice.

Remark 6. According to our knowledge, the paper is the first one investigating spread option pricing with stochastic interest rates. For stochastic interest rates, the study presents a more generalized spread option pricing model. Some models of stochastic interest rates are just a special case of the model presented by us. For Vasicek model (1), interest rates can easily go negative, which is a very bad property. Geman et al. [14] assumed that the volatility of the discounted bond price is a constant. For a random variable that is not going anywhere, sometimes the assumption is inappropriate. We assume that the volatility of the discounted bond price is a function of time $t$ rather than a constant.

Remark 7. The spread option pricing formula can be used in the standard call option. We present also a generalized Black-Scholes-Merton option pricing formula with stochastic interest rates. In fact, the price of the European call, assuming the risk-neutral dynamics given in (2) and (3) $(i=1)$, is $V(t)=V_{1}(t)+V_{3}(t)$, with

$$
\begin{aligned}
& V_{1}(t)=S_{1}(t) \operatorname{Pr}\left(1-K A_{31}(t) e^{B_{31}(t) \xi_{1}}>0\right), \\
& V_{3}(t)=-K B(t, T) \operatorname{Pr}\left(A_{13}(t) e^{B_{13}(t) \xi_{3}}-K>0\right),
\end{aligned}
$$

where the random variables $\xi_{1}$ and $\xi_{3}$ have the standard normal distribution. If the interest rate is a constant $r$, then $B(t, T)=e^{-r(T-t)}$; the result reduces to the usual BlackShcholes-Merton formula. The price of an exchange option (spread options with $K=0$ ), assuming the risk-neutral dynamics given in (2) and (3), is $V(t)=V_{1}(t)+V_{2}(t)+V_{3}(t)$ with

$$
\begin{aligned}
V_{1}(t)= & S_{1}(t) \operatorname{Pr}\left(1-A_{21}(t) e^{B_{21}(t) \xi_{1}}>0\right), \\
V_{2}(t)= & -S_{2}(t) \operatorname{Pr}\left(A_{12}(t) e^{B_{12}(t) \xi_{2}}-1>0\right), \\
V_{3}(t)= & -K B(t, T) \\
& \times \operatorname{Pr}\left(A_{13}(t) e^{B_{13}(t) \xi_{31}}-A_{23}(t) e^{B_{23}(t) \xi_{32}}>0\right),
\end{aligned}
$$

where the random variables $\xi_{1}$ and $\xi_{2}$ have the standard normal distribution and the random variables $\xi_{31}$ and $\xi_{32}$ have the bivariate standard normal distribution with correlation $\rho_{3}$.

Remark 8. The technique of measure changes is useful in a variety of asset pricing models. The risk-neutral valuation of the spread option price involves a two-dimensional integration rather than a three-dimensional integration. Monte Carlo approximations always contain some randomness. If we use some methods rather than Monte Carlo approximations, for the two-dimensional integration, we can increase the accuracy of the price or improve the computational efficiency. In particular, for the generalized Black-ScholesMerton option pricing formula with stochastic interest rates and the exchange option pricing formula in Remark 7, it is of great significance. In the cases, the risk-neutral valuations involve a one-dimensional integration rather than a twodimensional integration. Furthermore, by the technique of measure changes, the integrands become simple. As was pointed out in Carmona and Durrleman [1], even the most sophisticated models are very crude approximations of reality, and traders prefer having simple and reliable closed form formulae whose behavior can be well understood.

\section{Extension to Jump Diffusion Model}

This section constructs a spread option pricing model when underlying stock returns are discontinuous. As was pointed out in Merton (Ch. 9 in [18]), the abnormal vibrations in price are due to the arrival of important new information about the stock that has more than a marginal effect on price. This component is modeled by a jump process reflecting the nonmarginal impact of the information. In particular, a Poisson process is used to describe the arrival rate of catastrophe events and is applied for the pricing of CAT insurance products.

3.1. The Model. Let $(\Omega, \mathscr{F}, \widetilde{\mathbb{P}})$ be a probability space on which are defined a three-dimensional Brownian motion $\widetilde{W}(t)=$ $\left(\widetilde{W}_{1}(t), \widetilde{W}_{2}(t), \widetilde{W}_{3}(t)\right), 0 \leq t \leq T$, and two Poisson processes $N_{1}(t)$ and $N_{2}(t), 0 \leq t \leq T$, and $\widetilde{\mathbb{P}}$ denote the risk neutral probability measure. In particular, $\widetilde{W}_{a}(t), \widetilde{W}_{b}(t)$, and $\widetilde{W}_{c}(t)$ are independent Brownian motions. Let $\mathscr{F}(t), 0 \leq t \leq T$, be the filtration generated by the Brownian motion and the Poisson processes. Let $\lambda_{1}$ and $\lambda_{2}$ be the intensities of the Poisson processes $N_{1}(t)$ and $N_{2}(t)$, respectively. We define two compound Poisson processes as follows:

$$
Q_{i}(t)=\sum_{j=1}^{N_{i}(t)} Y_{i j}, \quad-1<X_{i j}, \quad i=1,2,
$$

where $Y_{i 1}, Y_{i 2}, \ldots$, random variables take values in the set $\left\{y_{i 1}, y_{i 2}, \ldots, y_{i M_{i 2}}\right\}$. The random variables $Y_{i 1}, Y_{i 2}, \ldots$, are independent and identically distributed, with $\mathbb{P}\left\{Y_{i j}=\right.$ $\left.y_{i m_{i}}\right\}=p\left(y_{i m_{i}}\right), m_{i}=1,2, \ldots, M_{i}$. We assume $\mathbb{E} Y_{i j}=$ $\sum_{m_{i}=1}^{M_{i}} y_{i m_{i}} p\left(y_{i m_{i}}\right)=\beta_{i}$. The evolutions of the discounted stock prices will be modeled by the stochastic differential equations:

$$
\begin{aligned}
d\left(D(t) S_{i}(t)\right)= & D(t) S_{i}(t) v_{i} \cdot d \widetilde{W}_{i}(t) \\
& +D(t) S_{i}(t-) d\left(Q_{i}(t)-\beta_{i} \lambda_{i} t\right), \quad i=1,2
\end{aligned}
$$

\subsection{Pricing the Spread Option}

Lemma 9. Under the measures $\widetilde{\mathbb{P}}^{S_{i}}, i=1,2$, the process $\widetilde{W}^{S_{i}}(t)=\left(\widetilde{W}_{a}(t), \widetilde{W}_{b}(t), \widetilde{W}_{c}(t)\right)-v_{i} t$ is a three-dimensional Brownian motion. $Q_{i}(t)$ is a compound Poisson process with 
intensity $\left(1+\beta_{i}\right) \lambda_{i}$ and independent, identically distributed jump sizes satisfying

$$
\begin{array}{r}
\widetilde{\mathbb{P}}^{S_{i}}\left\{Y_{i k}=y_{i m_{i}}\right\}=\frac{\left(1+y_{i m_{i}}\right) p\left(y_{i m_{i}}\right)}{1+\beta_{i}}, \\
k=1,2, \ldots, \quad m_{i}=1,2, \ldots, M_{i},
\end{array}
$$

and the processes $\widetilde{W}^{S_{i}}(t)$ and $Q_{i}(t)$ are independent.

The proof of Lemma 9 will be given in Appendix C.

Proposition 10. Under the following measures, the following price dynamics holds.

(1) Under the $T$-forward measure $\widetilde{\mathbb{P}}^{T}$, the process $S_{i}(t) / B(t$, $T), i=1,2$, is a martingale. Moreover,

$$
\begin{aligned}
& d\left(\frac{S_{i}(t)}{B(t, T)}\right) \\
& \quad=\frac{S_{i}(t-)}{B(t, T)}\left[\left(v_{i}-v_{3}(t, T)\right) \cdot d \widetilde{W}^{T}(t)+d\left(Q_{i}(t)-\beta_{i} \lambda_{i} t\right)\right],
\end{aligned}
$$

where $\widetilde{W}^{T}(t)=\left(\widetilde{W}_{a}(t), \widetilde{W}_{b}(t), \widetilde{W}_{c}(t)\right)-\int_{0}^{t} v_{3}(u, T) d u$ is a three-dimensional Brownian motion under $\widetilde{\mathbb{P}}^{T}$. The jump measure is unaffected by the change of measure.

(2) Under the measures $\widetilde{\mathbb{P}}^{S_{i}}, i=1,2$, the processes $B(t, T) / S_{i}(t)$ and $S_{j}(t) / S_{i}(t), j=1,2, i \neq j$, are $a$ martingale. Moreover,

$$
\begin{aligned}
& d\left(\frac{B(t, T)}{S_{i}(t)}\right)=\frac{B(t, T)}{S_{i}(t-)}\left[\left(v_{3}(t, T)-v_{i}\right) \cdot d \widetilde{W}^{S_{i}}(t)\right. \\
& \left.-d\left(\prod_{k=1}^{N_{i}(t)} \frac{Y_{i k}}{1+Y_{i k}}-\beta_{i} \lambda_{i} t\right)\right], \\
& d\left(\frac{S_{j}(t)}{S_{i}(t)}\right)=\frac{S_{j}(t-)}{S_{i}(t-)}\left[\left(v_{j}-v_{i}\right) \cdot d \widetilde{W}^{S_{i}}(t)\right. \\
& +d\left(Q_{j}(t)-\beta_{j} \lambda_{j} t\right) \\
& \left.-d\left(\prod_{k=1}^{N_{i}(t)} \frac{Y_{i k}}{1+Y_{i k}}-\beta_{i} \lambda_{i} t\right)\right]
\end{aligned}
$$

where $\widetilde{W}^{S_{i}}(t)=\left(\widetilde{W}_{a}(t), \widetilde{W}_{b}(t), \widetilde{W}_{c}(t)\right)-v_{i} t$ is a threedimensional Brownian motion under $\widetilde{\mathbb{P}}^{S_{i}}$.

The proof of Proposition 10 will be given in Appendix D.

By Proposition 10 and a similar discussion as the one in Theorem 5, we may obtain the following result.
Theorem 11. The price of the spread option, assuming the riskneutral dynamics given in (3) and (19), is

$$
\begin{gathered}
V(t)=\sum_{i=0}^{\infty} \sum_{j=0}^{\infty}\left[\sum _ { L = 1 } ^ { 2 } \widetilde { \mathbb { P } } ^ { S _ { L } } \left(N_{1}(T)-N_{1}(t)=i,\right.\right. \\
\left.N_{2}(T)-N_{2}(t)=j\right) S_{L}(t) V_{L}(t, i, j) \\
+\widetilde{\mathbb{P}}^{T}\left(N_{1}(T)-N_{1}(t)=i,\right. \\
\left.N_{2}(T)-N_{2}(t)=j\right) \\
\left.\times K B(t, T) V_{3}(t, i, j)\right]
\end{gathered}
$$

with

$$
\begin{aligned}
& V_{1}(t, i, j)=\widetilde{\mathbb{P}}^{S_{1}}\left(1-\prod_{k=1}^{i} \prod_{L=1}^{j} \frac{1+Y_{2 L}}{1+Y_{1 k}} A_{21}(t) e^{B_{21}(t) \xi_{11}}\right. \\
& \left.-\prod_{k=1}^{i} \frac{1}{1+Y_{1 k}} K A_{31}(t) e^{B_{31}(t) \xi_{12}}>0\right), \\
& V_{2}(t, i, j)=-\widetilde{\mathbb{P}}^{S_{2}}\left(\coprod_{k=1}^{i} \coprod_{L=1}^{j} \frac{1+Y_{1 k}}{1+Y_{2 L}} A_{12}(t) e^{B_{12}(t) \xi_{21}}-1\right. \\
& \left.-\prod_{L=1}^{j} \frac{1}{1+Y_{2 L}} K A_{32}(t) e^{B_{32}(t) \xi_{22}}>0\right), \\
& V_{3}(t, i, j)=-\widetilde{\mathbb{P}}^{T}\left(\prod_{k=1}^{i}\left(1+Y_{1 k}\right) A_{13}(t) e^{B_{13}(t) \xi_{31}}\right. \\
& -\prod_{L=1}^{j}\left(1+Y_{2 L}\right) A_{23} \\
& \left.\times(t) e^{B_{23}(t) \xi_{31}}-K>0\right)
\end{aligned}
$$

where

$$
\begin{gathered}
A_{i 3}(t)=\frac{S_{i}(t)}{B(t, T)} \exp \left\{-\frac{1}{2} \int_{t}^{T}\left\|\left(v_{i}-v_{3}(u, T)\right)\right\|^{2} d u\right. \\
\left.-\beta_{i} \lambda_{i}(T-t)\right\}, \\
\begin{aligned}
A_{3 i}(t)=\frac{B(t, T)}{S_{i}(t)} \exp \left\{-\frac{1}{2} \int_{t}^{T}\left\|\left(v_{3}(u, T)-v_{i}\right)\right\|^{2} d u\right. \\
\left.+\beta_{i} \lambda_{i}(T-t)\right\},
\end{aligned}
\end{gathered}
$$




$$
\begin{aligned}
A_{i j}(t)=\frac{S_{i}(t)}{S_{j}(t)} \exp \{ & -\frac{1}{2} \int_{t}^{T}\left\|\left(v_{i}-v_{j}\right)\right\|^{2} d u \\
& \left.+\beta_{i} \lambda_{i}(T-t)-\beta_{j} \lambda_{j}(T-t)\right\}, \\
& i, j=1,2, i \neq j .
\end{aligned}
$$

Remark 12. We can explore further applications of the formula beyond the case that the jump distribution is finitely many jump sizes. Suppose the jump sizes $y_{i j}, i=1,2$, $j=1,2, \ldots$, have a density $f_{i}\left(y_{i}\right)$ rather than a probability mass function $P\left(y_{i 1}\right), P\left(y_{i 2}\right), \ldots, P\left(y_{i M_{i}}\right)$. These densities are strictly positive on a set $B \subset(-1, \infty)$ and zero elsewhere. In the case $\beta_{i}=\int_{-1}^{\infty} f_{i}(x) d x$. By Lemma 9 and the definition of multiple integrals, we have that, under the measures $\widetilde{\mathbb{P}}^{S_{i}}$, $i=1,2$, the jumps in $Q_{i}(t)$ are independent and identically distributed with density $\left(1+y_{i}\right) f_{i}\left(y_{i}\right) /\left(1+\beta_{i}\right)$.

Remark 13. We can also apply the formula to a catastrophe option or European call in a jump model. The price of the European call, assuming the risk-neutral dynamics given in (3) and (19) $(i=1)$, is

$$
\begin{aligned}
V(t)=\sum_{i=0}^{\infty}\left[\widetilde{\mathbb{P}}^{S_{1}}\right. & \left(N_{1}(T)-N_{1}(t)=i\right) S_{1}(t) V_{1}(t, i) \\
+ & \widetilde{\mathbb{P}}^{T}\left(N_{1}(T)-N_{1}(t)=i\right) \\
& \left.\times K B(t, T) V_{3}(t, i, j)\right]
\end{aligned}
$$

where

$$
\begin{aligned}
& V_{1}(t, i) \\
& =\widetilde{\mathbb{P}}^{S_{1}}\left(1-\prod_{k=1}^{i} \frac{1}{1+Y_{1 k}} K A_{31}(t) e^{B_{31}(t) \xi_{12}}>0\right), \\
& V_{3}(t, i, j) \\
& \quad=-\widetilde{\mathbb{P}}^{T}\left(\prod_{k=1}^{i}\left(1+Y_{1 k}\right) A_{13}(t) e^{B_{13}(t) \xi_{31}}-K>0\right) .
\end{aligned}
$$

Remark 14. The technique of measure changes plays also a significant role for a variety of option pricing models when underlying stock returns are discontinuous. However, for the assumption of a constant interest rate, sometimes it is also useful. The European call in Remark 13 is a good example.

\section{Spread Option with Correlated Credit Risk and Stochastic Interest Rates}

The section constructs a spread option pricing model that allows not only for correlation between the option's underlying assets and the credit risk of the counterparty, but also for the interest rate to be random. For counterparty risk, we adopt some setting in the model of Klein [16].
The value of the asset of the counterparty $S_{4}(t)$ will be modeled by the stochastic differential equation:

$$
d\left(D(t) S_{4}(t)\right)=D(t) S_{i}(t) v_{4} \cdot d \widetilde{W}_{4}(t)
$$

where $\widetilde{W}_{4}(t)=\left(\widetilde{W}_{a}(t), \widetilde{W}_{b}(t), \widetilde{W}_{c}(t), \widetilde{W}_{d}(t)\right)$ and $v_{4}=\left(v_{41}, v_{42}\right.$, $\left.v_{43}, v_{44}\right)$. The value at time $t \in[0, T]$ of a spread option with counterparty risk, expiring at time $T$ with strike price $K$, is

$$
\begin{aligned}
& V(t)=\widetilde{\mathbb{E}}\left[e^{-\int_{t}^{T} R(u) d u}\left(S_{1}(T)-S_{2}(T)-K\right)^{+}\right. \\
& \times\left(\mathbb{q}_{\left\{S_{4}(T) \geq \gamma^{*}\right\}}+\mathbb{q}_{\left\{S_{4}(T)<\gamma^{*}\right\}}\right. \\
& \left.\left.\times \frac{(1-\alpha) S_{4}(T)}{\gamma}\right) \mid \mathscr{F}(t)\right] .
\end{aligned}
$$

At the exercise date $T$, a credit loss occurs if $S_{4}(T)$ is less than some amount $\gamma^{*}$. In the event of a credit loss, only the proportion $(1-\alpha) S_{4}(T) / \gamma$ of the nominal claim is paid out by the counterparty, where $\alpha$ represents the deadweight costs associated with bankruptcy expressed as a percentage of the value of the assets of the counterparty. $\gamma^{*}$ may be less than $\gamma$ due to the possibility of a counterparty continuing in operation even while $S_{4}(T)$ is less than $\gamma$. These deadweight costs include the direct cost of the bankruptcy or reorganization process, as well as the effects of distress on the business operations of the firm. If $S_{4}(T)$ is greater than $\gamma^{*}$ no credit loss occurs and the time $T$ claim receives full payment. We also refer the reader to the model of Klein [16] (a model of expected credit loss) for its relative discussion.

Theorem 15. The price of the spread option with correlated credit risk, assuming the risk-neutral dynamics given in (2), (3), and (28), is $V(t)=V_{1}(t)+V_{2}(t)+V_{3}(t)$ with

$$
\begin{aligned}
& V_{1}(t)=S_{1}(t) \mathbb{E}\left[\left(\bar{I}+A_{43}(t) e^{B_{43}(t) \xi_{3}} \underline{I}\right)\right. \\
& \left.\times \mathbb{q}_{\left\{1-A_{21}(t) e^{B_{21}(t) \xi_{11}}-K A_{31}(t) e^{B_{31}(t) \xi_{12}}>0\right\}}\right], \\
& V_{2}(t)=-S_{2}(t) \mathbb{E}\left[\left(\bar{I}+A_{43}(t) e^{B_{43}(t) \xi_{3}} \underline{I}\right)\right.
\end{aligned}
$$

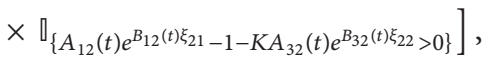

$$
\begin{aligned}
& V_{3}(t)=-K B(t, T) \mathbb{E}\left[\left(\bar{I}+A_{43}(t) e^{B_{43}(t) \xi_{3}} \underline{I}\right)\right. \\
& \left.\times \mathbb{I}_{\left\{A_{13}(t) e^{B_{13}(t) \xi_{31}}-A_{23}(t) e^{\left.B_{23}(t) \xi_{32}-K>0\right\}}\right.}\right],
\end{aligned}
$$

where

$$
\begin{aligned}
& A_{43}(t)=\frac{S_{4}(t)}{B(t, T)} \exp \left\{-\frac{1}{2} \int_{t}^{T}\left(\left\|v_{4}\right\|^{2}-\left\|v_{3}(u, T)\right\|^{2}\right) d u\right\}, \\
& B_{43}(t)=\left\|\sqrt{\int_{t}^{T}\left(v_{4}-v_{3}(u, T)\right)^{2} d u}\right\| . \\
& \bar{I}=\mathbb{\square}_{\left\{A_{43}(t) e^{B_{43}(t) \xi_{3}} \geq \gamma^{*}\right\}}, \quad \underline{I}=\mathbb{\square}_{\left\{A_{43}(t) e^{B_{43}(t) \xi_{3}}<\gamma^{*}\right\}} \text {. }
\end{aligned}
$$


The random variables $\xi_{3}$ and $\xi_{i 1}$ and $\xi_{3}$ and $\xi_{i 2}$ have the bivariate standard normal distribution with correlation $\widetilde{\rho_{i 1}}$ and $\widetilde{\rho_{i 2}}$, respectively, $i=1,2,3$, where

$$
\widetilde{\rho}_{k 1}=\frac{\left(v_{j}-v_{k}\right) \cdot \sqrt{\int_{t}^{T}\left(v_{4}-v_{3}(u, T)\right)^{2} d u}}{\left\|v_{j}-v_{k}\right\|\left\|\sqrt{\int_{t}^{T}\left(v_{4}-v_{3}(u, T)\right)^{2} d u}\right\|}
$$

$\widetilde{\rho}_{k 2}$

$$
=\frac{\sqrt{\int_{t}^{T}\left(v_{3}(u, T)-v_{k}\right)^{2} d u} \cdot \sqrt{\int_{t}^{T}\left(v_{4}-v_{3}(u, T)\right)^{2} d u}}{\left\|\sqrt{\int_{t}^{T}\left(v_{3}(u, T)-v_{k}\right)^{2} d u}\right\|\left\|\sqrt{\int_{t}^{T}\left(v_{4}-v_{3}(u, T)\right)^{2} d u}\right\|},
$$

$\tilde{\rho}_{3 L}$

$$
=\frac{\sqrt{\int_{t}^{T}\left(v_{L}-v_{3}(u, T)\right)^{2} d u} \cdot \sqrt{\int_{t}^{T}\left(v_{4}-v_{3}(u, T)\right)^{2} d u}}{\left\|\sqrt{\int_{t}^{T}\left(v_{L}-v_{3}(u, T)\right)^{2} d u}\right\|\left\|\sqrt{\int_{t}^{T}\left(v_{4}-v_{3}(u, T)\right)^{2} d u}\right\|},
$$$$
L=1,2 \text {. }
$$

Remark 16. Theorem 15 also presents a formula for a vulnerable Black-Scholes option with stochastic interest rates and correlated credit risk, which improves the result of the vulnerable Black-Scholes option in a model of Klein [16]. The price of a vulnerable Black-Scholes option, assuming the risk-neutral dynamics given in (2) $(i=1),(3)$, and (28), is $V(t)=V_{1}(t)+V_{3}(t)$ with

$$
\begin{aligned}
V_{1}(t)= & S_{1}(t) \mathbb{E} \\
& \times\left[\left(\bar{I}+A_{43}(t) e^{B_{43}(t) \xi_{3}} \underline{I}\right) \mathbb{q}_{\left\{1-K A_{31}(t) e^{\left.B_{31}(t) \xi_{12}>0\right\}}\right.}\right], \\
V_{3}(t)= & -K B(t, T) \mathbb{E} \\
& \times\left[\left(\bar{I}+A_{43}(t) e^{B_{43}(t) \xi_{3}} \underline{I}\right) \mathbb{q}_{\left\{A_{13}(t) e^{\left.B_{13}(t) \xi_{31}-K>0\right\}}\right.}\right] .
\end{aligned}
$$

\section{Numerical Experiments}

In this section, we use regular grid method (the area of each square of the grid is 0.000025 ) to the calculation of the formula when underlying stock returns are continuous and a mixture of both the regular grid method (the area of each square of the grid is 0.0001) and a Monte Carlo method (a random sample of 50000 for $N(T)-N(t) \geq 1$ ) to the one when underlying stock returns are discontinuous. We omit the numerical results for the spread option with credit risk to save space. However, they are available from the authors upon request.

Our modeling assumption is supplied by the following dynamics of the discounted price processes $D(t) S_{1}$ and $D(t) S_{2}$ and the short interest rate $R(t)$ :

$$
d\left(D(t) S_{1}(t)\right)=\sigma_{1} D(t) S_{1}(t) d \widetilde{W}_{a}(t),
$$

$$
\begin{aligned}
& d\left(D(t) S_{2}(t)\right)= \rho_{12} \sigma_{2} D(t) S_{2}(t) d \widetilde{W}_{a}(t) \\
&+\sqrt{1-\rho_{12}^{2}} \sigma_{2} D(t) B(t, T) d \widetilde{W}_{b}(t), \\
& d R(t)=\gamma(\tau-R(t)) d t-\sigma_{R} \rho_{13} d \widetilde{W}_{a}(t) \\
&-\sigma_{R} \eta_{1} d \widetilde{W}_{b}(t)-\sigma_{R} \eta_{2} d \widetilde{W}_{c}(t),
\end{aligned}
$$

where $\eta_{1}=\left(\rho_{23}-\rho_{12} \rho_{13}\right) / \sqrt{1-\rho_{12}^{2}}, \eta_{2}=\sqrt{1-\rho_{13}^{2}-\eta_{1}^{2}}$. We see that $\sigma_{i}^{2}, i=1,2$, is the instantaneous variance of the return on the underlying stock $S_{i}(t)$, and $\sigma_{R}$ is the instantaneous volatility of short-term interest rate. $\left(\widetilde{W}_{a}(t), \rho_{13} \widetilde{W}_{a}(t)+\right.$ $\left.\eta_{1} \widetilde{W}_{b}(t)+\eta_{2} \widetilde{W}_{c}(t)\right)$ and $\left(\rho_{12} \widetilde{W}_{a}(t)+\sqrt{1-\rho_{12}^{2}} \widetilde{W}_{b}(t), \rho_{13} \widetilde{W}_{a}(t)+\right.$ $\left.\eta_{1} \widetilde{W}_{b}(t)+\eta_{2} \widetilde{W}_{c}(t)\right)$ are a pair of correlated Brownian motions with correlation coefficients $\rho_{13}$ and $\rho_{23}$, respectively. $\gamma$ and $\tau$ are the respective constant reversion rate and long-term mean in the drift coefficient of the Vasicek dynamics of the interest rate. The price of the $T$-maturity zero coupon bond $B(t, T)$ is given by $B(t, T)=\exp (A(t, T)-C(t, T) R(t))$, where

$$
\begin{gathered}
A(t, T)=\left(\tau-\frac{\sigma_{R}^{2}}{2 \gamma^{2}}\right)(C(t, T)-(T-t))-\frac{\sigma_{R}^{2}}{4 \gamma} C^{2}(t, T), \\
C(t, T)=\frac{1}{\gamma}[1-\exp (-\gamma(T-t))],
\end{gathered}
$$

and the bond price satisfies the following:

$$
\begin{aligned}
d(B(t, T))= & B(t, T) R(t) d t+B(t, T) C(t, T) \sigma_{R} \rho_{13} d \widetilde{W}_{a}(t) \\
& +B(t, T) C(t, T) \sigma_{R} \eta_{1} d \widetilde{W}_{b}(t) \\
& +B(t, T) C(t, T) \sigma_{R} \eta_{2} d \widetilde{W}_{b}(t)
\end{aligned}
$$

Some parameters in Figures $1-4$ and Tables $1-3$ are fixed $(t=$ $0, T=1, \tau=5 \%, \gamma=0.3$ ).

Figure 1 shows how the price of the spread option varies with the changes in underlying asset prices $S_{1}(t)$ and $S_{2}(t)$. The remaining model parameters are $K=10, R(t)=1 \%$, $\sigma_{1}=20 \%, \sigma_{2}=20 \%, \sigma_{R}=15 \%, \rho_{12}=0.2, \rho_{13}=$ 0.2 , and $\rho_{23}=0.01$. Figure 2 shows how the price of the spread option varies with the changes in the volatilities $\sigma_{1}$ and $\sigma_{2}$. Larger volatilities induce more uncertainty and the option price increases as the volatility $\sigma_{1}$ or $\sigma_{2}$ increases. The remaining model parameters are $K=10, R(t)=1 \%$, $S_{1}=100, S_{2}=100, \sigma_{R}=15 \%, \rho_{12}=0.2, \rho_{13}=0.2$, and $\rho_{23}=0.01$. Figure 3 shows how the price of the spread option varies with the changes in the correlation coefficients $\rho_{12}$ or $\rho_{13}$. The changes in $\rho_{12}$ or $\rho_{13}$ significantly affect the value of the option. If the asset price of $S_{1}$ is strongly negatively correlated to interest rates and the asset price increases, then the probability that the option will be deep in the money at maturity date increases. The remaining model parameters are $K=10, R(t)=1 \%, S_{1}=100, S_{2}=100, \sigma_{1}=20 \%$, $\sigma_{2}=20 \%, \sigma_{R}=15 \%, \rho_{23}=0.01, \rho_{12}=0.2($ in Figure $3(\mathrm{~b})$ ), 


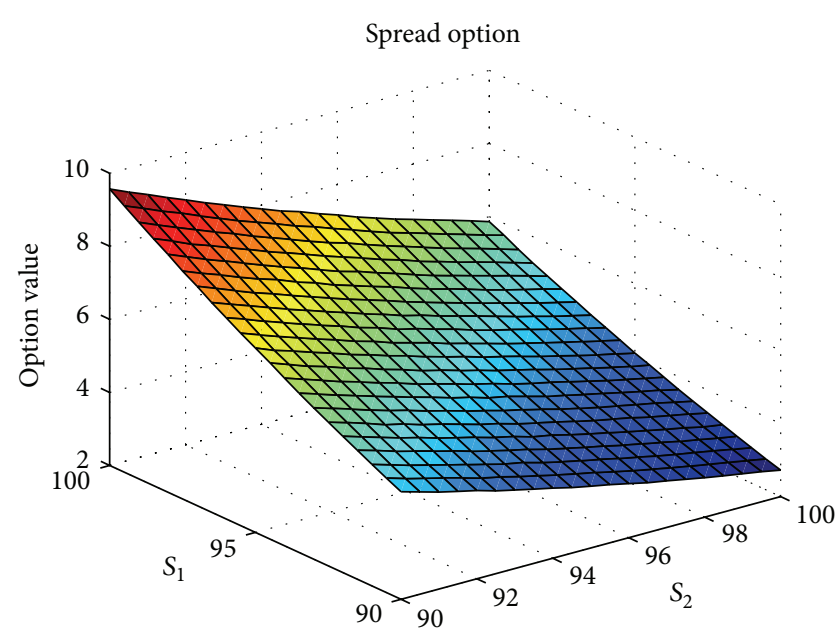

FIGURE 1: The price of the spread option varies with the changes in underlying asset prices $S_{1}(t)$ and $S_{2}(t)$.

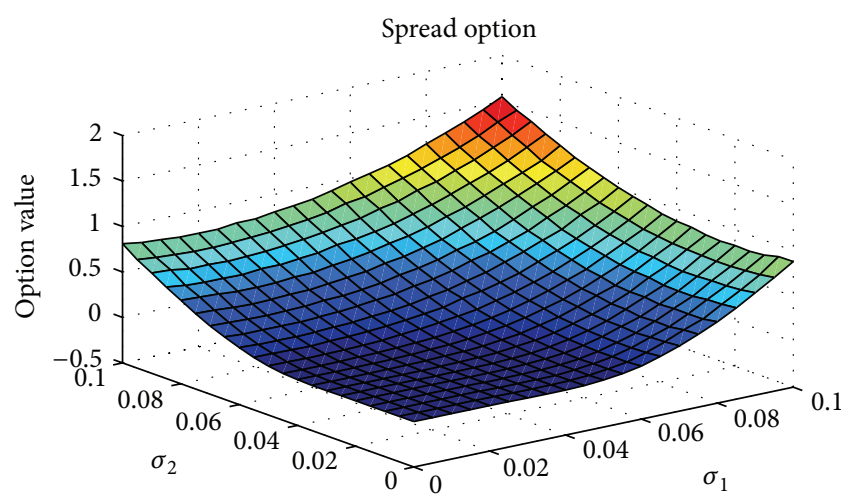

FIgURE 2: The price of the spread option varies with the changes in the volatilities $\sigma_{1}$ and $\sigma_{2}$.

and $\rho_{13}=0.2$ (in Figure 3(a)). Figure 4 shows how the price of the spread option varies with the changes in the spot interest rate $R(t)$ and the volatility $\sigma_{R}$. If the the spot interest rate $R(t)$ increases, then the price of the zero coupon $B(t, T)$ decreases, which increases the probability that the option becomes inthe-money. The remaining model parameters in Figures 4(a) and $4(\mathrm{~b})$ are $K=10, S_{1}=100, S_{2}=100, \sigma_{1}=20 \%, \sigma_{2}=20 \%$, $\sigma_{R}=15 \%, \rho_{12}=0.2, \rho_{13}=0.5$, and $\rho_{23}=0.01$ and $K=30$, $R(t)=5 \%, S_{1}=100, S_{2}=80, \sigma_{1}=20 \%, \sigma_{2}=20 \%, \rho_{12}=0.1$, $\rho_{13}=-0.5$, and $\rho_{23}=0.5$, respectively.

Now we show some numerical results for the spread option pricing in a jump model. All the following results are consistent with the behavior of the spread option price when the returns are discontinuous for all the two underlying stocks and, to simplify the discussion, we will focus on the cases $\lambda_{1} \neq 0$ and $\lambda_{2}=0$. We only assume $\left(\lambda_{2}=0\right)$ that the dynamics of the discounted price process $D(t) S_{1}$ is

$$
\begin{aligned}
d\left(D(t) S_{1}(t)\right)= & \sigma_{1} D(t) S_{1}(t) d \widetilde{W}_{a}(t) \\
& +D(t) S_{1}(t-) d\left(Q_{1}(t)-\beta_{1} \lambda_{1} t\right)
\end{aligned}
$$

TABLE 1: Spread option price.

\begin{tabular}{ccccccc}
\hline \multirow{K}{K}{} & \multicolumn{5}{c}{$\lambda_{1}$} \\
& 0 & 0.04 & 0.08 & 0.12 & 0.16 & 0.2 \\
\hline 2 & 9.0963 & 9.3481 & 9.5591 & 9.7337 & 9.8761 & 9.9900 \\
4 & 8.1906 & 8.5078 & 8.7794 & 9.0103 & 9.2046 & 9.3664 \\
6 & 7.3595 & 7.6421 & 7.8821 & 8.0840 & 8.2522 & 8.3903 \\
8 & 6.5753 & 6.8796 & 7.1398 & 7.3606 & 7.5464 & 7.7009 \\
10 & 5.8556 & 6.1613 & 6.4232 & 6.6459 & 6.8336 & 6.9901 \\
\hline
\end{tabular}

TABLE 2: Spread option price.

\begin{tabular}{lcccccc}
\hline \multirow{2}{\rho_{12}}{} & \multicolumn{6}{c}{$\lambda_{1}$} \\
& 0 & 0.04 & 0.08 & 0.12 & 0.16 & 0.2 \\
\hline-0.8 & 10.5947 & 10.8515 & 11.0598 & 11.2249 & 11.3516 & 11.4443 \\
-0.6 & 9.7783 & 10.0964 & 10.3634 & 10.5845 & 10.7648 & 10.9087 \\
-0.4 & 8.9031 & 9.1473 & 9.3474 & 9.5083 & 9.6344 & 9.7297 \\
-0.2 & 7.9746 & 8.2249 & 8.4324 & 8.6018 & 8.7374 & 8.8429 \\
0 & 6.9684 & 7.2559 & 7.4994 & 7.7034 & 7.8722 & 8.0098 \\
0.2 & 5.8622 & 6.1258 & 6.3488 & 6.5356 & 6.6902 & 6.8161 \\
0.4 & 4.6314 & 4.9373 & 5.2013 & 5.4276 & 5.6203 & 5.7830 \\
0.6 & 3.2155 & 3.5425 & 3.8272 & 4.0741 & 4.2872 & 4.4702 \\
0.8 & 1.5161 & 1.8841 & 2.2092 & 2.4958 & 2.7479 & 2.9691 \\
\hline
\end{tabular}

TABLE 3: Spread option price.

\begin{tabular}{lcccccc}
\hline$\rho_{13}$ & 0 & 0.04 & 0.08 & 0.12 & 0.16 & 0.2 \\
\hline-0.8 & 6.0963 & 6.4456 & 6.7721 & 7.0777 & 7.3642 & 7.6332 \\
-0.6 & 6.0512 & 6.3431 & 6.6131 & 6.8632 & 7.0952 & 7.3110 \\
-0.4 & 6.0086 & 6.3172 & 6.5990 & 6.8566 & 7.0924 & 7.3086 \\
-0.2 & 5.9608 & 6.2466 & 6.5026 & 6.7319 & 6.9372 & 7.1210 \\
0 & 5.9153 & 6.2018 & 6.4529 & 6.6722 & 6.8632 & 7.0287 \\
0.2 & 5.8629 & 6.1612 & 6.4163 & 6.6325 & 6.8140 & 6.9644 \\
0.4 & 5.8057 & 6.0576 & 6.2611 & 6.4216 & 6.5440 & 6.6328 \\
0.6 & 5.7472 & 6.0189 & 6.2281 & 6.3818 & 6.4862 & 6.5469 \\
0.8 & 5.6825 & 5.9355 & 6.1092 & 6.2126 & 6.2539 & 6.3406 \\
\hline & & & & & &
\end{tabular}

where $Y_{11}, Y_{12}, \ldots$, have a density $f_{1}\left(y_{1}\right) \approx$ $\sqrt{2 \pi} 0.4) \exp \left(-y_{1}^{2} / 0.32\right)$ for $-1<y_{1}<1$.

Table 1 shows how the price of the spread option varies with the changes in the exercise price $K$ and the Poisson active rate $\lambda_{1}$. Larger intensity induces more uncertainty and the option price increases as the Poisson active rate increases. The remaining model parameters are $R(t)=1 \%, S_{1}=100$, $S_{2}=100, \sigma_{1}=20 \%, \sigma_{2}=20 \%, \sigma_{R}=15 \%, \rho_{12}=0.2$, $\rho_{13}=0.2$, and $\rho_{23}=0.01$. Table 2 shows how the price of the spread option varies with the changes in the correlation coefficient $\rho_{12}$ and the Poisson active rate $\lambda_{1}$. The remaining model parameters are $K=10, R(t)=1 \%, S_{1}=100, S_{2}=100$, $\sigma_{2}=20 \%, \sigma_{R}=15 \%, \rho_{13}=0.2$, and $\rho_{23}=0.01$. Table 3 shows how the price of the spread option varies with the changes in the correlation coefficient $\rho_{13}$ and the Poisson active rate $\lambda_{1}$. The remaining model parameters are $K=10, R(t)=1 \%$, $S_{1}=100, S_{2}=100, \sigma_{2}=20 \%, \sigma_{R}=15 \%, \rho_{12}=0.2$, and $\rho_{23}=0.01$. For $K, \rho_{12}$, and $\rho_{13}$, the results in Tables $1-3$ are 


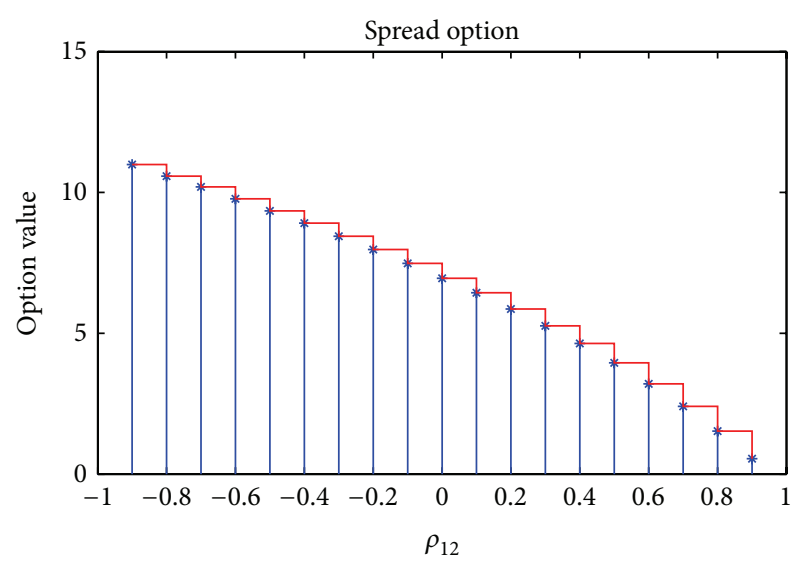

(a)

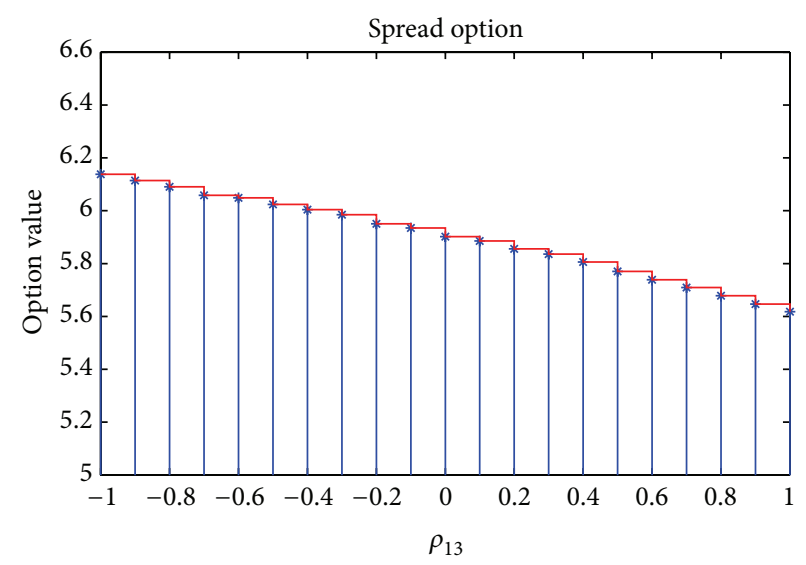

(b)

FIGURE 3: The price of the spread option varies with the changes in the correlation coefficients $\rho_{12}$ or $\rho_{13}$.

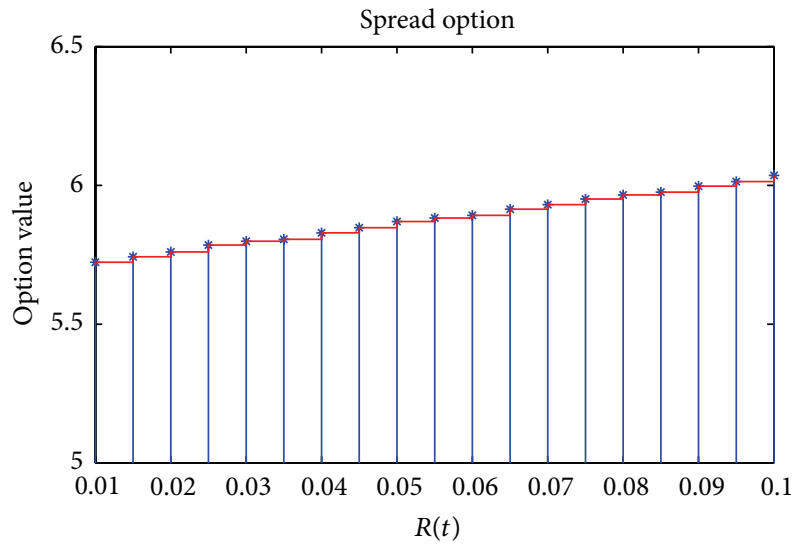

(a)

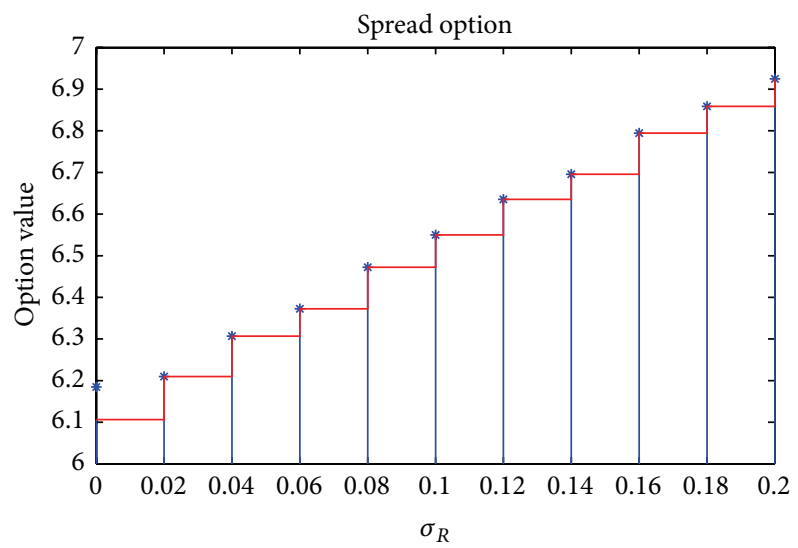

(b)

FIGURE 4: The price of the spread option varies with the changes in the spot interest rate $R(t)$ and the volatility $\sigma_{R}$.

consistent with the behavior of the spread option price when underlying stock returns are continuous.

\section{Concluding Remarks}

In this paper, we have explored further analysis of spread option pricing beyond the case that the interest rate is a constant. The model of stochastic interest rates and the technique of measure changes are useful in a variety of asset pricing models. Through numerical experiments and simulations, we demonstrated that the changes in correlation coefficients, the volatilities of the returns on underlying stocks, and the Poisson active rate significantly affect the price, and stochastic interest rates play a significant role. In general, existing methods for pricing spread options can be divided into two groups: numerical methods and analytical approximations. Analytical approximation methods are generally faster than numerical methods but often lack accuracy and robustness. For the spread option pricing formula (without counterparty risk) when underlying stock returns are continuous, by the technique of measure changes, the integrand becomes simple. In the case, the method is both accurate and fast (by regular grid method, it is easy to derive suitable accurate price). By a mixture of both the regular grid method and a Monte Carlo method to the calculation of the formula when underlying stock returns are discontinuous, we can obtain a suitable approximate price.

\section{Appendices}

\section{A. Proof of Proposition 4}

By (2) and (3), we have

$$
\begin{aligned}
\frac{B(t, T)}{S_{i}(t)}=\frac{B(0, T)}{S_{i}(0)} \exp \{ & -v_{i} \cdot \widetilde{W}_{i}(t)+v_{3}(t, T) \cdot d \widetilde{W}_{3}(t) \\
& \left.+\frac{1}{2}\left\|v_{i}\right\|^{2} t-\frac{1}{2}\left\|v_{3}(t, T)\right\|^{2} t\right\} .
\end{aligned}
$$


It follows from (A.1) and Itô's formula that

$$
\begin{aligned}
d\left(\frac{B(t, T)}{S_{i}(t)}\right)=\frac{B(t, T)}{S_{i}(t)}\{ & -v_{i} \cdot d \widetilde{W}_{i}(t)+v_{3}(t, T) \cdot d \widetilde{W}_{3}(t) \\
& +\frac{1}{2}\left\|v_{i}\right\|^{2} d t-\frac{1}{2}\left\|v_{3}(t, T)\right\|^{2} d t \\
& \left.+\frac{1}{2}\left\|v_{i}-v_{3}(t, T)\right\|^{2} d t\right\} \\
= & \frac{B(t, T)}{S_{i}(t-)}\left(v_{3}(t, T)-v_{i}\right) \cdot d \widetilde{W}^{S_{i}}(t)
\end{aligned}
$$

where $\widetilde{W}^{S_{i}}(t)=\left(\widetilde{W}_{a}(t), \widetilde{W}_{b}(t), \widetilde{W}_{c}(t)\right)-v_{i} t$. By Lemma 2 , Definition 1, and (2), we have that $\widetilde{W}^{S_{i}}(t)$ is a three-dimensional Brownian motion under $\widetilde{\mathbb{P}}^{S_{i}}$. Using the fact that $\widetilde{W}^{S_{i}}(t)$ is a three-dimensional Brownian motion under $\widetilde{\mathbb{P}}^{S_{i}}$ and Lemma 3, we may obtain that the process $B(t, T) / S_{i}(t)$ is a martingale under the measures $\mathbb{P}^{S_{i}}$. By a similar approach as the one used in the above case, we can obtain the remaining results in Proposition 4.

\section{B. Proof of Theorem 5}

We prove the result for $t=0$. It is not difficult to modify the proof of account for general $t$. At time zero, the value of the option expiring at time $T$ is $V(0)=V_{1}(0)+V_{2}(0)+V_{3}(0)$, where

$$
\begin{gathered}
V_{i}(0)=\widetilde{\mathbb{E}}\left[D(T) S_{i}(T) \mathbb{q}_{\left\{S_{1}(T)-S_{2}(T)-K>0\right\}}\right], \quad i=1,2, \\
V_{3}(0)=-K B(0, T) \widetilde{\mathbb{E}}\left[D(T) \mathbb{q}_{\left\{S_{1}(T)-S_{2}(T)-K>0\right\}}\right] .
\end{gathered}
$$

By Definition 1 , we may rewrite $V_{1}(0), V_{2}(0)$, and $V_{3}(0)$ as

$$
\begin{aligned}
V_{i}(0) & =S_{i}(0) \widetilde{\mathbb{P}}^{S_{i}}\left\{\frac{S_{1}(T)}{S_{i}(T)}-\frac{S_{2}(T)}{S_{i}(T)}-\frac{K}{S_{i}(T)}>0\right\}, \\
i & =1,2, \\
V_{3}(0)= & -K B(0, T) \widetilde{\mathbb{P}}^{T} \\
& \times\left\{\frac{S_{1}(T)}{B(T, T)}-\frac{S_{2}(T)}{B(T, T)}-\frac{K}{B(T, T)}>0\right\} .
\end{aligned}
$$

Put (12), (B.2), and Proposition 4 together; we may obtain the result of Theorem 5 .

\section{Proof of Lemma 9}

Let $N_{i}^{(k)}$ denote the number of jumps in $Q_{i}$ of size $x_{m_{i}}$ up to and including time $t$. Then

$$
N_{i}(t)=\sum_{m_{i}=1}^{M_{i}} N_{i}^{\left(m_{i}\right)}(t), \quad Q_{i}(t)=\sum_{m_{i}=1}^{M_{i}} x_{m_{i}} N_{i}^{\left(m_{i}\right)}(t) .
$$

The processes $N_{i}^{(1)}, N_{i}^{(2)}, \ldots, N_{i}^{\left(M_{i}\right)}$ defined this way are independent Poisson processes, and each $N_{i}^{\left(m_{i}\right)}$ has intensity $\lambda_{i} p\left(y_{i m_{i}}\right)$. By (19) and (C.1), we may obtain

$$
\begin{aligned}
D(t) S_{i}(t)= & S_{i}(0) \exp \left\{v_{i} \cdot \widetilde{W}_{i}(t)-\frac{1}{2}\left\|v_{i}\right\|^{2} t\right\} \\
& \times \prod_{m_{i}=1}^{M_{i}} e^{\left(\lambda_{i} p\left(x_{m_{i}}\right)-\left(1+y_{i m_{i}}\right) \lambda_{i} p\left(y_{i m_{i}}\right) t\right.} \\
& \times\left(\frac{\left(1+y_{i m_{i}}\right) \lambda_{i} p\left(y_{i m_{i}}\right)}{\lambda_{i} p\left(y_{i m_{i}}\right)}\right)^{N_{i}^{\left(m_{i}\right)}(t)} .
\end{aligned}
$$

By (C.2) and the fact that the processes $N_{i}^{(1)}, N_{i}^{(2)}, \ldots, N_{i}^{\left(M_{i}\right)}$ are independent Poisson processes, we may obtain the results of Lemma 9.

\section{Proof of Proposition 10}

By (3) and (19), we have

$$
\begin{aligned}
& \frac{B(t, T)}{S_{i}(t)} \\
& \quad=\frac{B(0, T)}{S_{i}(0)} \exp \left\{-v_{i} \cdot \widetilde{W}_{i}(t)+v_{3}(t, T) \cdot d \widetilde{W}_{3}(t)\right. \\
& \left.\quad+\frac{1}{2}\left\|v_{i}\right\|^{2} t-\frac{1}{2}\left\|v_{3}(t, T)\right\|^{2} t+\beta_{i} \lambda_{i} t\right\} \\
& \quad \times \prod_{k=1}^{N_{i}(t)} \frac{1}{1+Y_{i k}} .
\end{aligned}
$$

It follows from (D.1) and Itô's formula for one jump process that

$$
\begin{aligned}
d\left(\frac{B(t, T)}{S_{i}(t)}\right)=\frac{B(t, T)}{S_{i}(t)}\{ & -v_{i} \cdot d \widetilde{W}_{i}(t)+v_{3}(t, T) \cdot d \widetilde{W}_{3}(t) \\
& +\frac{1}{2}\left\|v_{i}\right\|^{2} d t-\frac{1}{2}\left\|v_{3}(t, T)\right\|^{2} d t \\
& \left.+\frac{1}{2}\left\|v_{i}-v_{3}(t, T)\right\|^{2} d t+\beta_{i} \lambda_{i} d t\right\} \\
& -\frac{B(t, T)}{S_{i}(t-)} d\left(\prod_{k=1}^{N_{i}(t)} \frac{Y_{i k}}{1+Y_{i k}}\right) \\
=\frac{B(t, T)}{S_{i}(t-)}\left[\left(v_{3}(t, T)-v_{i}\right) \cdot d \widetilde{W}^{S_{i}}(t)\right. & \left.-d\left(\prod_{k=1}^{N_{i}(t)} \frac{Y_{i k}}{1+Y_{i k}}-\beta_{i} \lambda_{i} t\right)\right]
\end{aligned}
$$

where $\widetilde{W}^{S_{i}}(t)=\left(\widetilde{W}_{a}(t), \widetilde{W}_{b}(t), \widetilde{W}_{c}(t)\right)-v_{i} t$ is a three-dimensional Brownian motion under $\widetilde{\mathbb{P}}^{S_{i}}$. According to (20), we 
have $\widetilde{\mathbb{E}}^{S_{i}}\left[Y_{i k} /\left(1+Y_{i k}\right)\right]=\sum_{m_{i}=1}^{M_{i}}\left(y_{i m_{i}}\left(1+y_{i m_{i}}\right) p\left(y_{i m_{i}}\right) /(1+\right.$ $\left.\left.y_{i m_{i}}\right)\left(1+\beta_{i}\right)\right)=\beta_{i} /\left(1+\beta_{i}\right), i=1,2, \ldots$. Then, using the fact that $Q_{i}(t)$ is a compound Poisson process with intensity $\left(1+\beta_{i}\right) \lambda_{i}$, we may obtain

$$
\widetilde{\mathbb{E}}^{S_{i}}\left(\prod_{k=1}^{N_{i}(t)} \frac{Y_{i k}}{1+Y_{i k}}\right)=\beta_{i} \lambda_{i} t .
$$

By (D.2) and (D.3), we have that the process $B(t, T) / S_{i}(t)$ is a martingale under the measure $\mathbb{P}^{S_{i}}$. By a similar approach as the one used in the above case, we can obtain the remaining results in Proposition 10.

\section{Conflict of Interests}

The authors declare that there is no conflict of interests regarding the publication of this paper.

\section{Acknowledgment}

This work was supported by the National Natural Science Foundation of China (61273015).

\section{References}

[1] R. Carmona and V. Durrleman, "Pricing and hedging spread options," SIAM Review, vol. 45, no. 4, pp. 627-685, 2003.

[2] T. R. Hurd and Z. Zhou, "A Fourier transform method for spread option pricing," SIAM Journal on Financial Mathematics, vol. 1, no. 1, pp. 142-157, 2010.

[3] R. Caldana and G. Fusai, "A general closed-form spread option pricing formula," Journal of Banking and Finance, vol. 37, no. 12, pp. 4893-4906, 2013.

[4] P. Bjerksund and G. Stensland, "Closed form spread option valuation,” Quantitative Finance, vol. 14, no. 10, pp. 1785-1794, 2011.

[5] C. F. Lo, "A simple derivation of Kirk's approximation for spread options," Applied Mathematics Letters, vol. 26, no. 8, pp. 904907, 2013.

[6] N. D. Pearson, "An effcient approach for pricing spread options," Journal of Derivatives, vol. 3, pp. 76-91, 1995.

[7] P. Laurence and T. Wang, "Distribution-free upper bounds for spread options and market-implied antimonotonicity gap," European Journal of Finance, vol. 14, no. 8, pp. 717-734, 2008.

[8] S.-J. Deng, M. Li, and J. Zhou, "Closed-form approximations for spread option prices and Greeks," Journal of Derivatives, vol. 15, no. 3, pp. 58-80, 2008.

[9] M. A. H. Dempster, E. Medova, and K. Tang, "Long term spread option valuation and hedging," Journal of Banking and Finance, vol. 32, no. 12, pp. 2530-2540, 2008.

[10] S. E. Shreve, Stochastic Calculus for Finance II: Continuous-Time Models, Springer, New York, NY, USA, 2004.

[11] Y.-K. Kwok, Mathematical Models of Financial Derivatives, Springer, 2nd edition, 2008.

[12] R. C. Merton, "Theory of rational option pricing," Bell Journal of Economics and Management Science, vol. 4, pp. 141-183, 1973.

[13] F. Jamshidian, "An exact bond option formula," The Journal of Finance, vol. 44, pp. 205-209, 1989.

[14] H. Geman, N. E. Karoui, and J. Rochet, "Changes of numeraire, change of probability measure and option pricing," Journal of Applied Probability, vol. 32, no. 2, pp. 443-458, 1995.
[15] J. Hull, Options, Futures and Other Derivations Securities, Pearson Education, 8th edition, 2012.

[16] P. Klein, "Pricing black-scholes options with correlated credit risk," Journal of Banking \& Finance, vol. 20, no. 7, pp. 1211-1229, 1996.

[17] S.-J. Yang, M.-K. Lee, and J.-H. Kim, "Pricing vulnerable options under a stochastic volatility model," Applied Mathematics Letters, vol. 34, pp. 7-12, 2014.

[18] R. C. Merton, Continuous Time Finance, Wiley-Blackwell, 1992. 


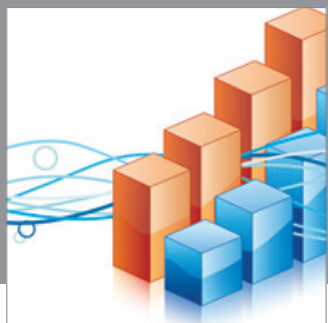

Advances in

Operations Research

mansans

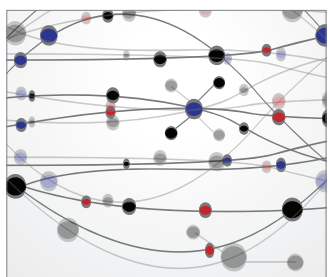

The Scientific World Journal
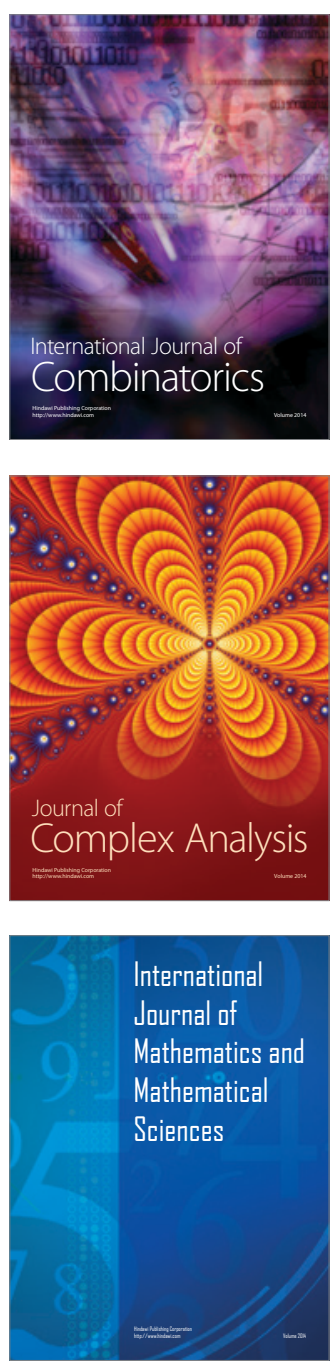
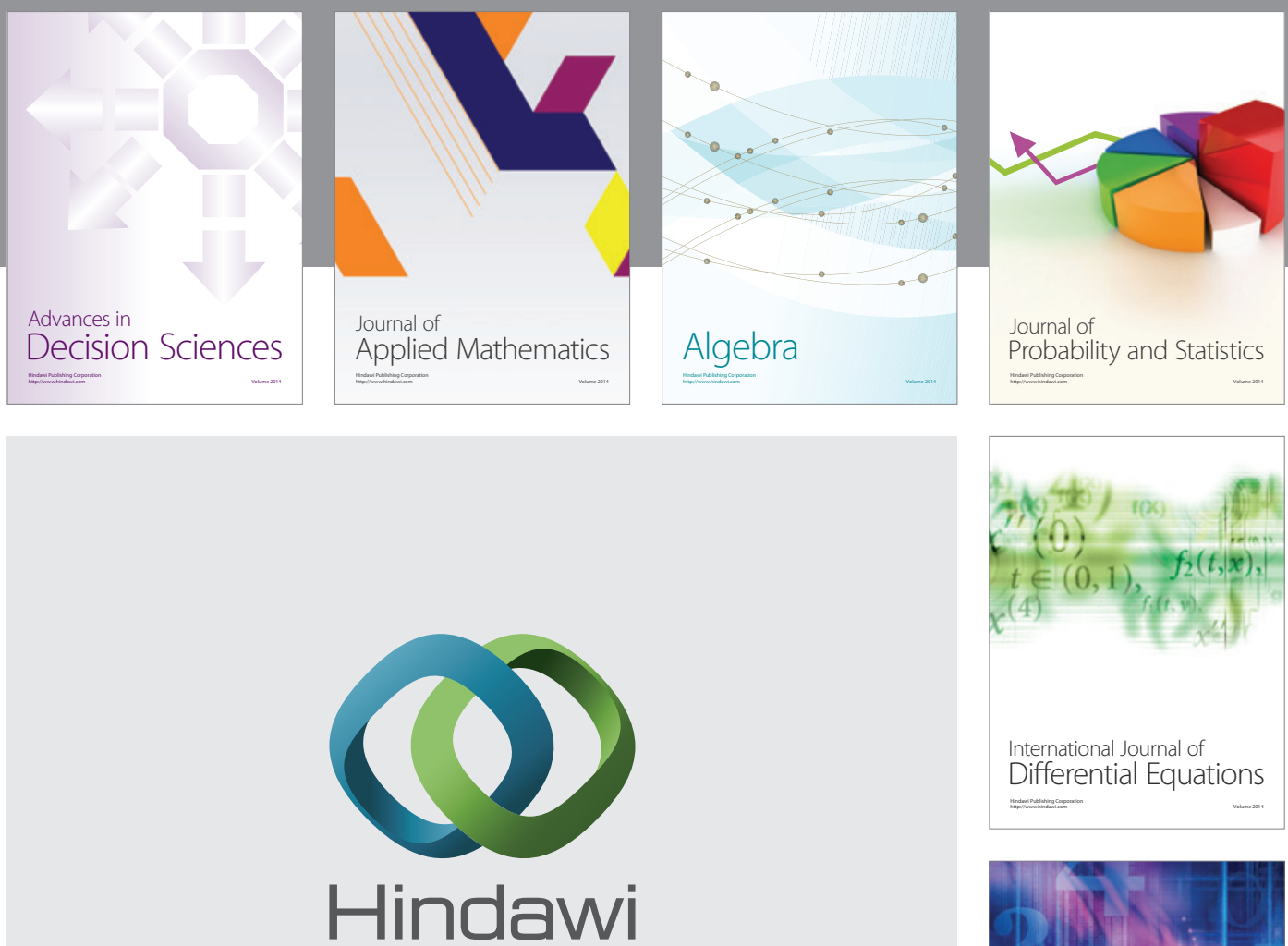

Submit your manuscripts at http://www.hindawi.com
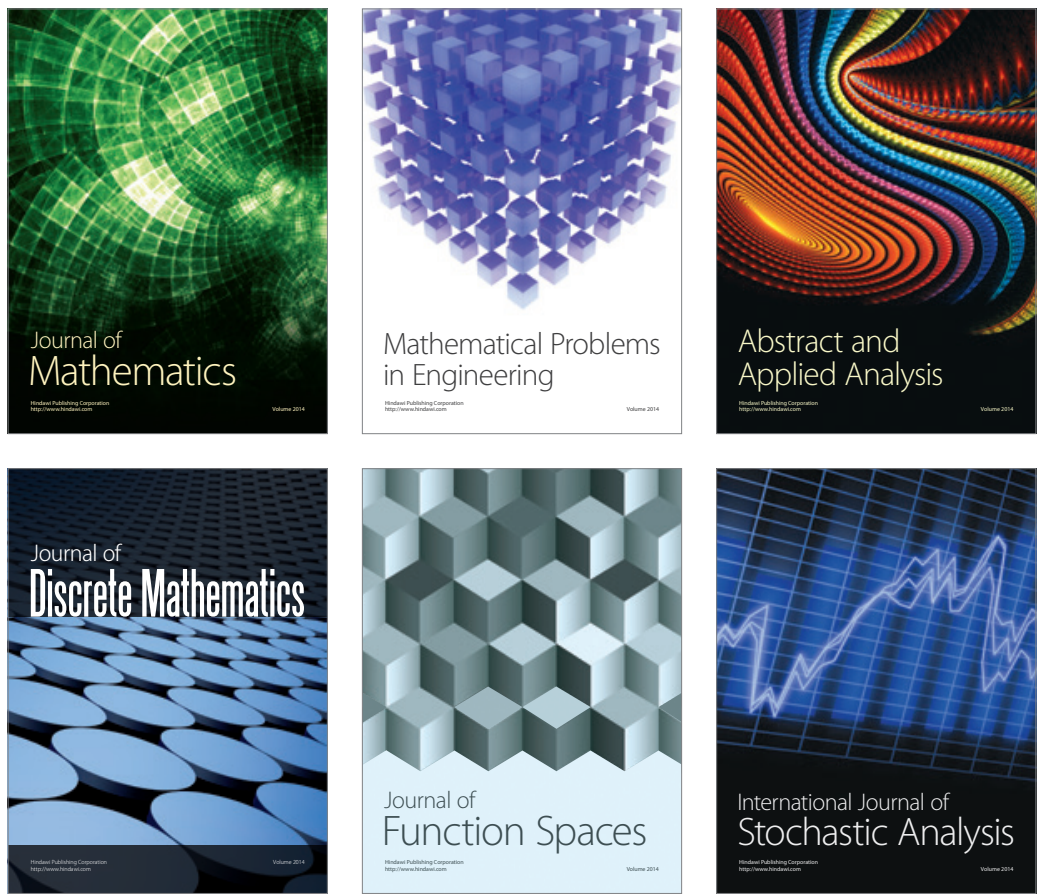

Journal of

Function Spaces

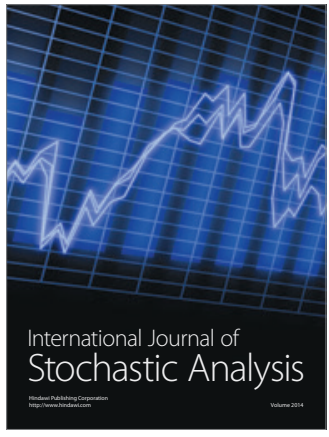

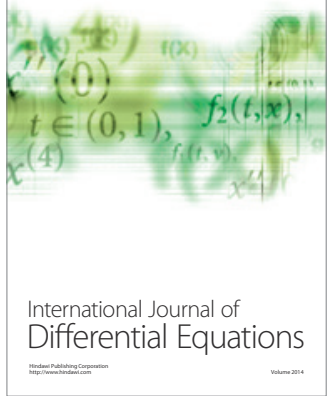
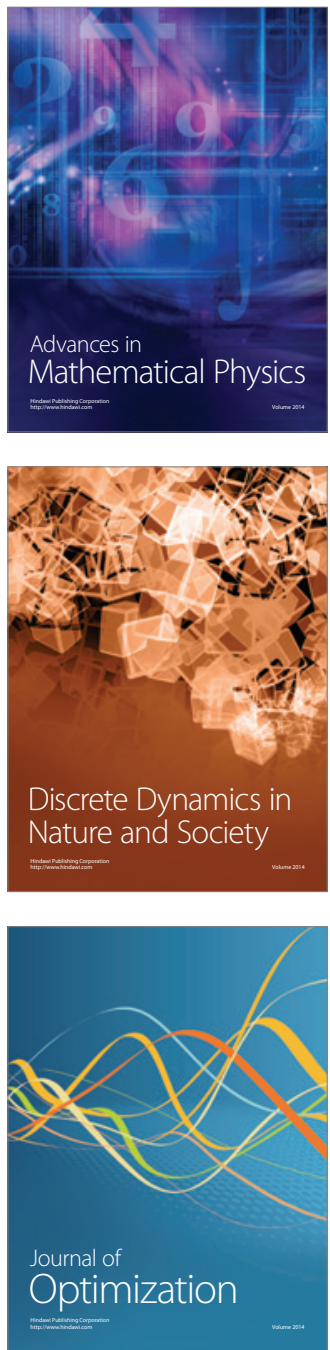